\title{
Phylogeny and evolution of the family Ectothiorhodospiraceae based on comparison of 16S rRNA, cbbL and nifH gene sequences
} Correspondence
Tatjana P. Tourova
ttour@biengi.ac.ru

\section{INTRODUCTION}

The family Ectothiorhodospiraceae forms a separate phylogenetic lineage within the Gammaproteobacteria according to 16S rRNA gene sequence analysis and mostly includes halophilic and haloalkaliphilic bacteria. Originally, this family was created to accommodate purple sulfur bacteria that deposit elemental sulfur outside the cell. Later, it was divided into the moderately halo(alkali)philic species of the genus Ectothiorhodospira and the extremely halo(alkali)philic species of the genus Halorhodospira (Imhoff \&

Abbreviations: RubisCO, ribulose-1,5-bisphosphate carboxylase/oxygenase; RSCU, relative synonymous codon usage.

The GenBank/EMBL/DDBJ accession numbers for the sequences reported in this paper are EF199939-EF199958 and EF202525.
Süling, 1996). More recently, two new genera, Thiorhodospira (Bryantseva et al., 1999) and Ectothiorhodosinus (Gorlenko et al., 2004), made up of moderately haloalkaliphilic purple sulfur bacteria isolated from soda lakes, were added to the family. All these phototrophic bacteria prefer to grow anaerobically in the light using reduced sulfur compounds as electron donors. At the same time, representatives of several genera with aerobic chemotrophic metabolism are closely related to the anaerobic phototrophs of the Ectothiorhodospiraceae, namely the heterotrophs Arhodomonas (Adkins et al., 1993) and Alkalispirillum (Rijkenberg et al., 2001), the facultative autotroph Alkalilimnicola (Yakimov et al., 2001; Sorokin et al., 2006; Hoeft et al., 2007), the obligately chemolithoautotrophic nitrifier Nitrococcus (Watson \& Waterbury, 1971; Teske et al., 1994) and chemolithotrophic sulfur-oxidizing 
bacteria of the genera Thioalkalivibrio (Sorokin et al., 2001, 2002a, b; Banciu et al., 2004) and Thioalkalispira (Sorokin et al., 2002c). This raises several interesting questions on the evolution of the family.

The modern taxonomy of the Ectothiorhodospiraceae follows their phylogenetic relationships based on 16S rRNA gene sequences (Fig. 1). The gene encoding the $16 \mathrm{~S}$ rRNA is widely used as a universal molecular marker for phylogenetic reconstructions and taxonomy because it has been assumed that intraspecies variation and horizontal transfer of this gene were low. Some housekeeping protein-encoding genes, e.g. gyrB, recA and $r p o B$ (Dauga, 2002; Holmes et al., 2004), have been used as additional molecular markers for phylogenetic studies of different bacterial groups. Phylogenetic reconstructions based on sequence analyses of the 16S rRNA gene and housekeeping protein-encoding genes usually correlate quite well, but sometimes an additional analysis of functional genes is necessary in order to clarify uncertain cases. The functional genes responsible for key metabolic properties can also be used as alternative molecular markers. Sequence analysis of functional genes might help to resolve difficult taxonomic problems as well as to clarify the evolution of corresponding metabolic pathways.

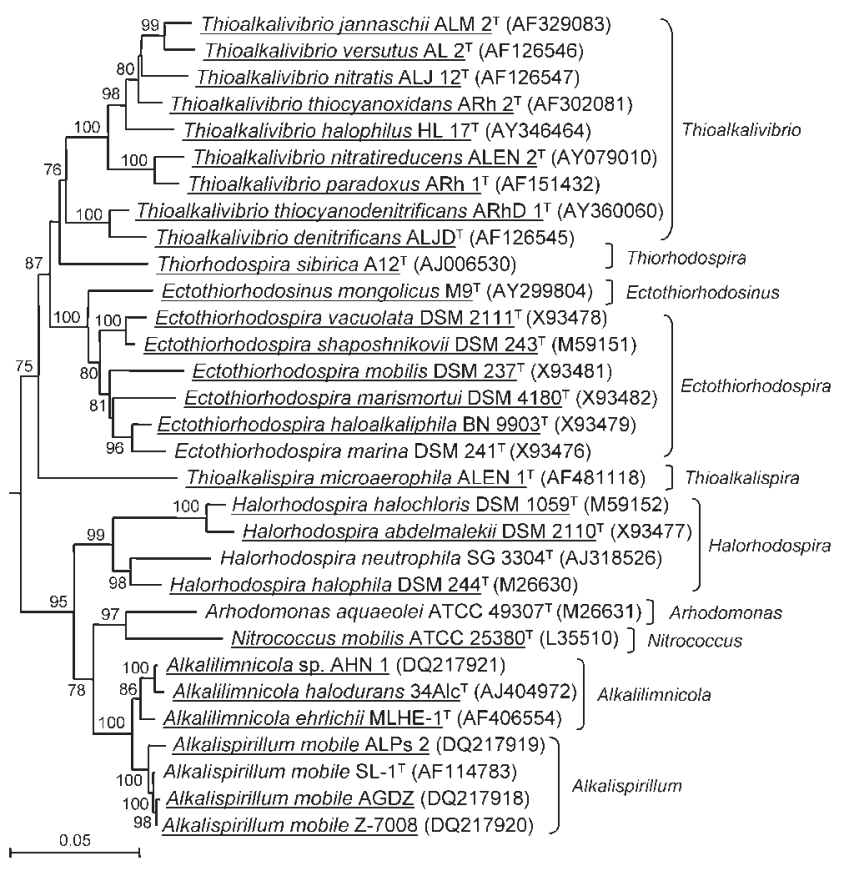

Fig. 1. Phylogeny of the family Ectothiorhodospiraceae based on analyses of 16S rRNA gene sequences. Strains for which RubisCO gene sequences were determined are underlined. The tree topography and evolutionary distances are given by the neighbour-joining method with Jukes and Cantor distances. Numbers at nodes indicate percentage bootstrap values for the clade of this group in 1000 replications. Only values above $75 \%$ were considered significant. Bar, $5 \%$ sequence divergence.
Most representatives of the family Ectothiorhodospiraceae are obligate or facultative autotrophs. It has been shown that phototrophic species of Ectothiorhodospira and Halorhodospira (Imhoff, 2006) and chemolithotrophic species of Thioalkalivibrio (Sorokin et al., 2001, 2002a, b; Banciu et al., 2004) and Thioalkalispira (Sorokin et al., 2002c) have ribulose-1,5-bisphosphate carboxylase/oxygenase (RubisCO) activity. RubisCO is the key enzyme of the Calvin-Benson-Bassham reductive pentose phosphate cycle. Two different RubisCO forms are known. The large catalytic subunit of form I is encoded by $c b b L$, while the only subunit of form II is encoded by $c b b M$. The $c b b L$ gene is in turn divided into 'green-like' and 'red-like' types. The 'green-like' $c b b L$ gene has recently been found in the genomes of Ectothiorhodospira shaposhnikovii (Spiridonova et al., 2004), Thioalkalivibrio species (Tourova et al., 2005) and the Alkalispirillum-Alkalilimnicola group (Oremland et al., 2002; Sorokin et al., 2006).

The capacity for nitrogen fixation has been shown for most purple phototrophic bacteria, including some members of the Ectothiorhodospiraceae (Moshkovskii et al., 1971; Imhoff, 2005). During the process of biological nitrogen fixation, the enzyme nitrogenase catalyses the ATPdependent reduction of dinitrogen to ammonia. Nitrogenase consists of two component metalloproteins, the iron $(\mathrm{Fe})$ protein (encoded by nifH) and the molybdenum-iron $(\mathrm{MoFe})$ protein (encoded by nifD and nifK). The Fe protein mediates the coupling of ATP hydrolysis to interprotein electron transfer, and its gene is highly conserved among closely related micro-organisms. This means that nifH can be used to study relationships among diazotrophic bacteria. Nevertheless, the occurrence and phylogeny of nifH in representatives of the Ectothiorhodospiraceae has not yet been investigated. The only exception is Halorhodospira halophila strain BN 9629, where the nitrogenase operon has recently been cloned and characterized (Tsuihiji et al., 2006).

The aim of the present study was to extend the evolutionary analysis of the family Ectothiorhodospiraceae based on the comparison of the 16S rRNA gene-based phylogeny with phylogenies of genes encoding the key functional enzymes RubisCO ( $c b b L)$ and nitrogenase (nifH).

\section{METHODS}

Bacterial strains. Strains used in this study are listed in Table 1. Type strains of the genera Alkalispirillum and Arhodomonas were obtained from the DSMZ. Nitrococcus mobilis ATCC $25380^{\mathrm{T}}$ was kindly provided by Dr E. Spieck (Universität Hamburg, Germany). Biomass of these bacteria was used directly for DNA extraction. Other strains were from the culture collections of the Institute of Microbiology and of the Department of Microbiology of Moscow State University (KM MGU). The cultures were maintained as described previously (Imhoff, 2005; Bryantseva et al., 1999; Sorokin et al., 2001, 2002a, b, c).

DNA isolation and PCR amplification. DNA extraction and purification were performed as described previously (Boulygina 
Table 1. Comparison between the $G+C$ contents (mol\%) of the cbbL and nifH genes and the full genome in the family Ectothiorhodospiraceae

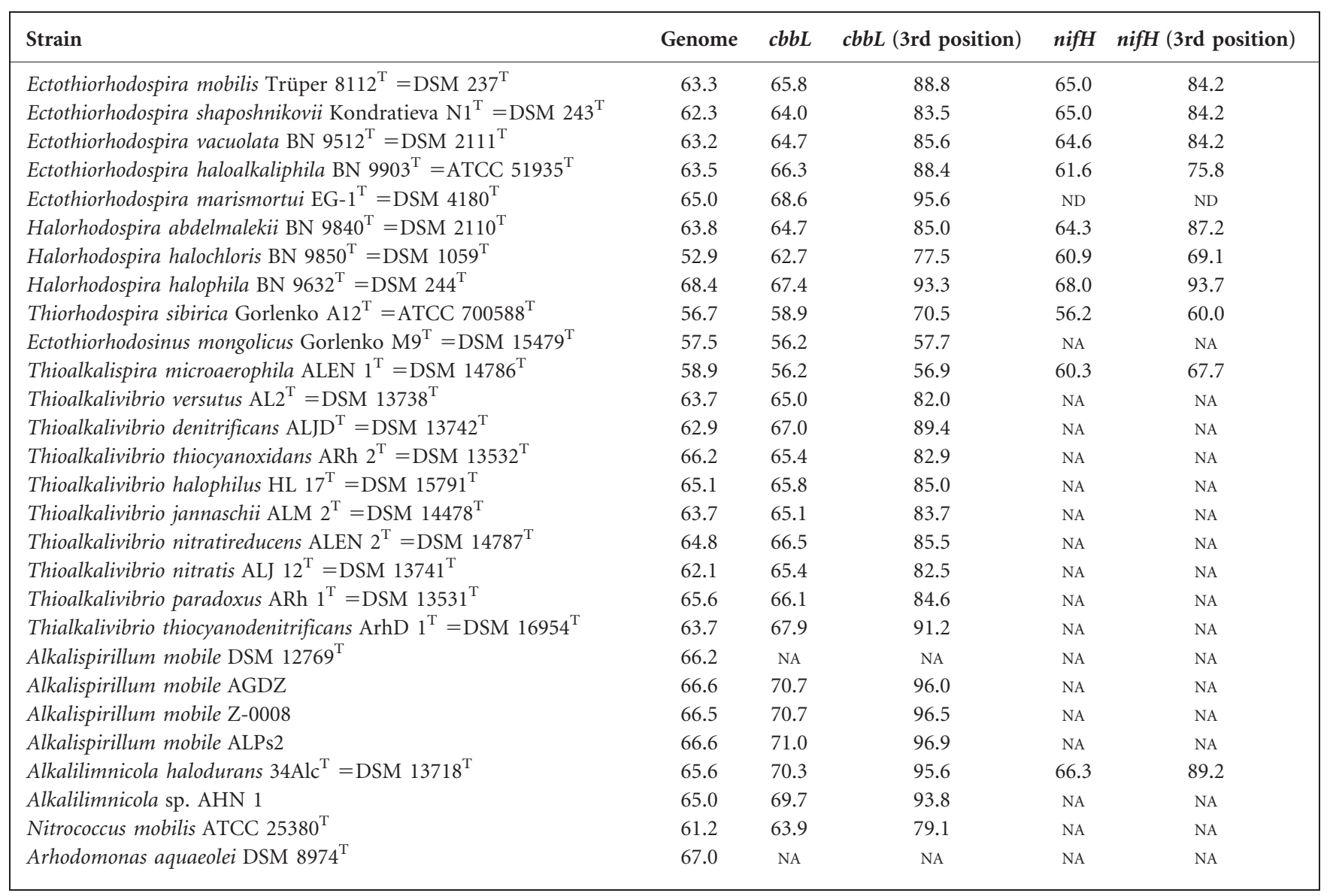

ND, Not determined; NA, not applicable.

et al., 2002). The $c b b L$ and nifH gene fragments were amplified using specially developed and previously tested primers (Spiridonova et al., 2004; Marusina et al., 2001). PCR products were purified from lowmelting-point agarose using the Wizard PCR Preps kit (Promega).

Cloning and sequencing of the PCR fragments. Purified PCR products were cloned using the pGEM-T vector system (Promega). Plasmid DNA was extracted and purified using the Wizard MiniPrep kit (Promega). Clones containing appropriately sized inserts were sequenced from universal M13 forward and reverse primers (Sambrook et al., 1989). Sequencing was performed with an ABI 3730 sequencer using the Big Dye Terminator v. 3.1 sequencing reaction kit (Applied Biosystems).

Phylogenetic analysis. Preliminary analysis of the new sequences was performed via the NCBI BLAST server (http://www.ncbi.nlm.nih. gov/BLAST/). Nucleotide and inferred amino acid sequences were aligned with sequences from GenBank using CLUSTAL w (Thompson et al., 1994).

Genetic distances were calculated using Kimura's two-parameter method (Kimura, 1980). To obtain synonymous and non-synonymous distances, a method of Nei \& Gojobori (1986) was applied to the various sequences of protein-encoding genes using WET software (J. Dopazo; http://www.tdi.es). Phylogenetic trees were reconstructed using four different algorithms: neighbour-joining (Saitou \& Nei,
1987) in the TREECONW program package (Van de Peer \& De Wachter, 1994) and maximum-parsimony (Fitch, 1971), distance matrix (Fitch \& Margoliash, 1967) and maximum-likelihood (Felsenstein, 1981) using PHYLIP 3.5c software (Felsenstein, 1993). Relative synonymous codon usage (RSCU) values of the $c b b L$ and nifH genes were calculated using CodonW software (J. Peden; http://codonw.sourceforge.net). To investigate the major codon usage trends in different species, CodonW was used to carry out a correspondent analysis. Each gene produced a point in the codon space, the positions of which suggested a possible codon usage bias.

\section{RESULTS}

\section{Detection and phylogenetic analysis of Rubisco genes}

Primers specific to the genes encoding 'red-like' form I and form II of RubisCO did not amplify corresponding gene fragments in any of the investigated strains listed in Table 1. On the other hand, using the specific primer set for the 'green-like' $c b b L$ gene, PCR products of about 750 bp were obtained from the DNAs of all strains, except the type strains of Alkalispirillum mobile and Arhodomonas 
aquaeolei. Clones prepared from the $c b b L$ PCR fragments yielded a single sequence-type for all species. Results of BLAST analysis revealed a high level of similarity between the newly determined nucleotide sequences and the other $c b b L$ sequences available in GenBank, confirming their affiliation to the same family of genes.

The nucleotide sequences obtained for RubisCO gene fragments as well as the deduced amino acid sequences of the corresponding proteins were aligned with analogous sequences of the 'green-like' $c b b L$ from GenBank. Positions with gaps and ambiguous sequences were removed and the remaining 720 nucleotide and 240 amino acid positions were used for further phylogenetic analysis. The topologies of the phylogenetic trees constructed on the basis of these alignments were similar for all the methods used, the neighbour-joining (Fig. 2), maximum-parsimony, distancematrix and maximum-likelihood (data not shown) methods.

Similar to results published previously (Watson \& Tabita, 1997), the topologies obtained did not correspond to those of the 'ribosomal' tree. According to $16 \mathrm{~S}$ rRNA gene sequence analysis, all members of the family Ectothiorhodospiraceae form a monophyletic clade within the Gammaproteobacteria, consisting of several phylogenetic clusters and branches corresponding to different genera (Fig. 1). However, the $c b b L$-based trees suggest that the Ectothiorhodospiraceae is divided into independent clusters and branches with positions in the trees dependent on the set of chosen reference sequences (Fig. 2). These intrafamily divisions correlated only partially with the $16 \mathrm{~S}$ rRNA gene-based tree topology. Different species of the genera Halorhodospira and Alkalispirillum-Alkalilimnicola formed monophyletic clusters with high bootstrap values (100 and 98-100\%, respectively, based on nucleotides and amino acids), and the monotypic genus Thioalkalispira formed a separate branch unrelated to other members of the family in both trees. However, the genus Ectothiorhodospira realistically formed a single cluster (with $91 \%$ bootstrap value) in the 'RubisCO' tree based on nucleotides only, whereas in the tree based on amino acids this cluster was unstable (31\% bootstrap value), depending on the set of chosen reference sequences. Moreover, in the 'RubisCO' trees, the monotypic genus Ectothiorhodosinus formed a separate branch unrelated to Ectothiorhodospira species.

The most dramatic discrepancies between the topologies of the 'ribosomal' and 'RubisCO' trees were observed in the case of the genus Thioalkalivibrio. According to the RubisCO gene analysis, Thioalkalivibrio is not a monophyletic genus (Tourova et al., 2005). The Thioalkalivibrio species formed three independent clusters in the nucleotide-based 'RubisCO' tree (with 74-100\% bootstrap values) and two clusters (34-100\% bootstrap values) and one branch in the amino acid-based tree. This division correlated only partially with the inner structure of the single 'ribosomal' Thioalkalivibrio cluster. Only the cluster combining Thioalkalivibrio denitrificans and Thioalkalivibrio thiocyanodenitrificans (the deepest Thioalkalivibrio subcluster according to the 'ribosomal' tree) demonstrated a distant relationship to Ectothiorhodospira in the nucleotide-based 'RubisCO' tree ( $75 \%$ bootstrap value). At the same time, the phylogenetic position was strongly supported in both 'RubisCO' trees only for the cluster Thioalkalivibrio nitratireducens/ Thioalkalivibrio paradoxus. This cluster grouped with Nitrococcus mobilis with high bootstrap values (98 and $100 \%$, respectively, based on nucleotides and amino acids) and with one of the duplicated $c b b L$ genes of Allochromatium vinosum (with 100 and $70 \%$ bootstrap values, respectively, for nucleotide and amino-acid-based trees). The position of Thiorhodospira sibirica in both RubisCO trees was unexpected, because it grouped together with some representatives of the Alphaproteobacteria with strong bootstrap support (respectively 98 and $97 \%$ based on nucleotides and amino acids).

\section{Detection and phylogenetic analysis of nitrogenase reductase genes}

PCR products of the expected size (about $450 \mathrm{bp}$ ) were obtained with the nifH-specific primers using the DNAs of all investigated species of the phototrophic genera Ectothiorhodospira, Halorhodospira and Thiorhodospira, but not of the genus Ectothiorhodosinus. Unexpectedly, nifH fragments were also obtained for the chemotrophic species Thioalkalispira microaerophila and Alkalilimnicola halodurans, for which diazotrophic potential was not suspected previously. Preliminary screening in the GenBank database demonstrated that all newly determined nucleotide sequences belong to the nifH gene family. Moreover, the nifH gene fragment obtained from the type strain of $\mathrm{Hl}$. halophila was almost identical to the analogous fragment of the previously sequenced nifH gene from Hlr. halophila BN 9629 (GenBank accession no. AB189641).

The nucleotide sequences of nifH gene fragments as well as the deduced amino acid sequences of the corresponding proteins were aligned, positions with gaps and ambiguous sequences were removed and the remaining 444 nucleotide and 148 amino acid positions were used for further phylogenetic analysis. The topologies of the phylogenetic trees constructed on the basis of these alignments were similar for all methods used: the neighbour-joining (Fig. 3), maximum-parsimony, distance-matrix and maximumlikelihood (data not shown) methods.

The topologies of the nifH-based trees correlated only partially with the 'ribosomal' tree. Similar to the $c b b L$ based trees, species of the family Ectothiorhodospiraceae did not form a monophyletic clade. Moreover, in the case of nifH, the Ectothiorhodospira species (as well as Halorhodospira representatives) were clustered together only in the nucleotide-based tree (with 55 and $96 \%$ bootstrap values, respectively). On the other hand, the nifH-based phylogeny of the monotypic genus Thiorhodospira demonstrated a clear relationship of this bacterium to Ectothiorhodospira mobilis, Ectothiorhodospira shaposhnikovii and Ectothiorhodospira vacuolata ( $80 \%$ bootstrap value for the 
Ectothiorhodospira marismortui DSM 4180 ${ }^{\top}$ (EF199942)

Thioalkalivibrio thiocyanodenitrificans ARhD1 ${ }^{\top}$ (AY914802)

Thioalkalivibrio denitrificans ALJD' (AY914807)

- Acidifhiobactllus ferrooxidans ATCC $23270^{\top} \mathrm{cbbL}-2$ (AF307091)

Allochromatium vinosum ATCC $17899^{\top}$ rbCA (M26396)

0.1

$100-$ Thioalkalivibrio jannaschii ALM2 $^{\top}$ (AY914803)

7 Thioalkalivibrio nitratis A $\mathrm{J} 12^{\mathrm{T}}$ (AY014805)

Thioakalivibrio versutus AL2T (AY914800)

Thioalkalivibrio thiocyanoxidans ARh $2^{\top}$ (AY914804)

Alkalispirillum mobile Z-7008 (DQ217925)

75 Alkalispirillum mabile AGDZ (DQ217922)

100 Alkaliimnicola sp AHN 1 (DQ217923)

100 Alkalilimnicola halodurans 34 Alc $^{\top}$ (DQ217926) Alkalilimnicola halodurans $34 \mathrm{Alc}^{\top}{ }^{\top}$ (DQ217920)

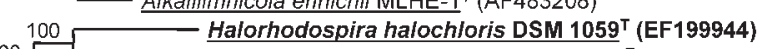
Halorhodospira abdelmalekii DSM 2110 ${ }^{\top}$ (EF19994) Halorhodospira halophila DSM $244^{\top}$ (EF199945)
Thioclava pacifica TL $2^{\top}$ (AY656720)

99 Thiorhodospira sibirica A12 ${ }^{\top}$ (EF199948) Rhodobacter capsulatus SB1003 (L82000)

Rhodovulum sulfidophilum DSM 1374 (AY656721)

80 $100-$ Allochromatium vinosum ATCC 17899' $\mathrm{rbCL}$ (D90204) Nitrococcus mobilis ATCC 25380 (EF1

Thioalkalivibrio nitratireducens ALEN 2 ${ }^{\top}$ (AY914801)

$100-$ Nitrosospira sp. TCH716 (AF459718) Nitrosomonas sp. ENI-11 (AB061373)

100 Nitrobacter winogradskyi ATCC 14123 (AF109914) - Nitrobacter vulgaris T3 (L22885)

Ectothiorhodosinus mongolicum M9' (EF199947)

Acidithiobacillus ferrooxidans ATCC

Hydrogenovibrio marinus $\mathrm{MH}-110^{\top}$ cbbL-1 (D43621)

Hydrogenovibrio marinus $\mathrm{MH}-110^{\top} \mathrm{CbbL}-2$ (D43622)

(b) $81-$ Thioalkalivibrio nitratis AL $J 12^{\top}$ (AY914805)

Thioalkalivibrio versutus AL2 ${ }^{\top}$ (AY914800)

Thioalkalivibrio thiocyanoxidans ARh $2^{\top}$ (AY914804)

94 Thioalkalivibrio halophilus HL 17 ${ }^{\top}$ (EF199949)

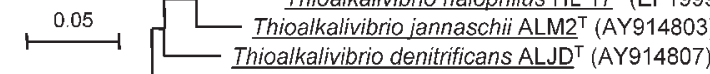

Ectothiorhodospira haloalkaliphila BN 9903 ${ }^{\top}$ (EF199941)

- Ectothiorhodospira marismortui DSM 4180 ${ }^{\top}$ (EF199942)

Ectothiorhodospira shaposhnikovii DSM 243 ${ }^{\top}$ (AY450587)

${ }_{85} \leftarrow$ Ectothiorhodospira mobilis DSM $237^{\top}$ (EF199939)

$100-$ Halorhodospira halochloris DSM $1059^{\top}$ (EF199944)

$100-$ Halorhodospira halochloris DSM $1059^{\top}$ (EF199944)

Halorhodospira halophila DSM 244 ${ }^{\top}$ (EF199945)

- Allochromatium vinosum ATCC $17899^{\top}$ rbCA (M26396)

Ectothiorhodosinus mongolicus M9 ${ }^{\top}$ (EF199947)

Nitrosomonas sp. ENI-11 (AB061373)

100 Nitrobacter winogradskyi ATCC 14123 (AF109914)

- Nitrobacter vulgaris T3 (L22885)

Acidithiobacillus ferrooxidans ATCC $23270^{\top}$ cbbL-2 (AF307091)

Thioalkalivibrio thiocyanodenitrificans $\mathrm{ARhD}^{\top}{ }^{\top}$ (AY914802)
Hydrogenovibrio marinus $\mathrm{MH}-110^{\top} \mathrm{cbbL}-1$
(D43621)

98 Thiociava pacifica TL $2^{\top}$ (AY656720)

Rhodobacter capsulatus SB1003 (L82000)

- Rhodovulum sulfidophilum DSM 1374 (AY656721)

Acidithiobacillus ferrooxidans ATCC 23270 T cbbL-1 (AF129925)

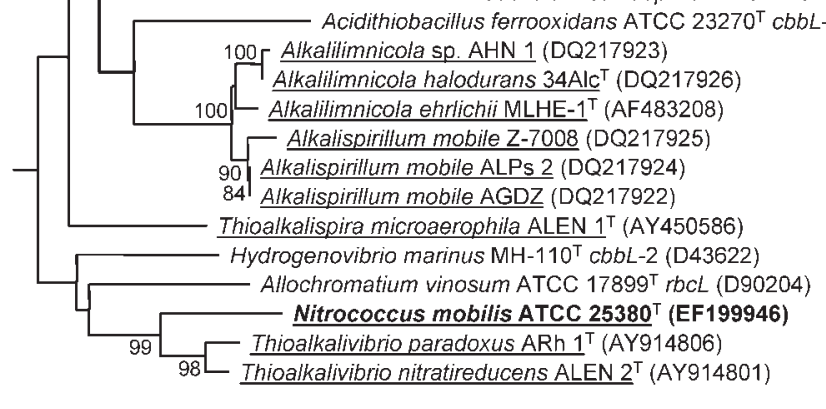

Fig. 2. Phylogenetic positions of species of the Ectothiorhodospiraceae in $c b b L$ molecular trees based on analysis of nucleotide sequences (a) and translated amino acid sequences (b). Sequences of species of the Ectothiorhodospiraceae determined in this study are marked in bold. Underlining marks sequences belonging to species of the family Ectothiorhodospiraceae. Tree topography and evolutionary distances are given by the neighbour-joining method with Jukes and Cantor (for nucleotides) and Poisson (for amino acids) corrections. Numbers at nodes indicate percentage bootstrap values for the clade of this group in 1000 replications. Only values above $75 \%$ were considered significant. Bars, $10 \%$ (a) or $5 \%$ (b) sequence divergence. 

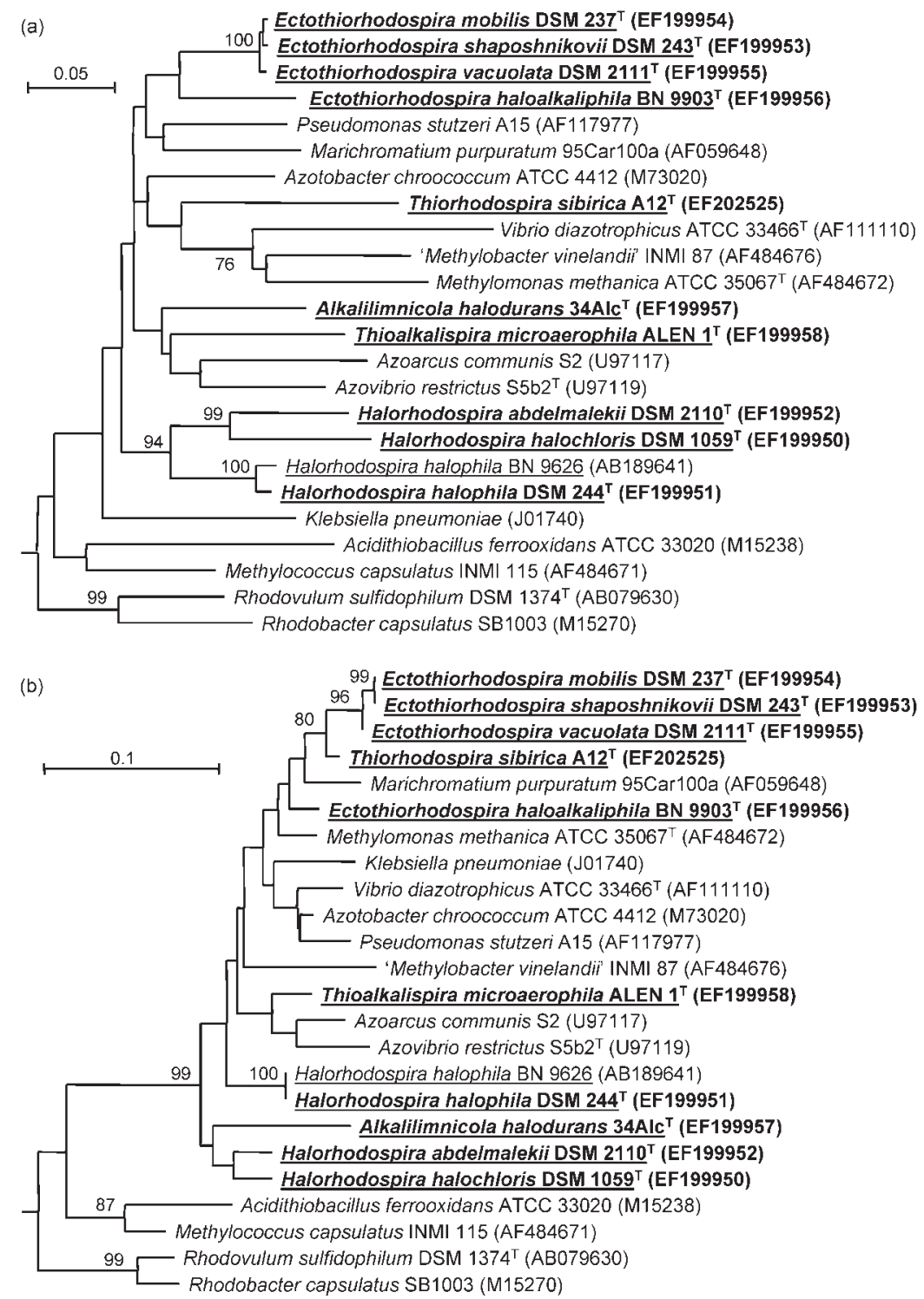

Fig. 3. Phylogenetic position of species of the Ectothiorhodospiraceae in nifH molecular trees based on analysis of nucleotide sequences (a) and translated amino acid sequences (b). Sequences determined in this study are marked in bold. Underlining marks sequences belonging to species of the family Ectothiorhodospiraceae. See the legend to Fig. 2 for further details. Bars, $5 \%$ (a) or $10 \%$ (b) sequence divergence. amino-acid-based tree) and was similar to the $16 \mathrm{~S}$ rRNA gene-based phylogeny, although it differed from the $c b b L$ based one.

The nifH phylogeny of Thioalkalispira microaerophila and Alkalilimnicola halodurans was of particular interest. In both 'nitrogenase' trees, they formed two separate branches with an uncertain branching point position, and were slightly related only to Azoarcus and Azovibrio, representing the Betaproteobacteria (bootstrap values did not exceed 56\%). Thus, their nifH genes do not have a clear relationship to the nifH of the other members of the Ectothiorhodospiraceae.

\section{Comparison of genetic distances among the $c b b L$, nifH and 16S rRNA genes}

Nucleotide substitutions within protein-encoding regions are divided into two classes: synonymous (silent), which are largely invisible to natural selection, and nonsynonymous (resulting in amino acid replacement), which may be under strong selective pressure. Synonymous distances in the $c b b L$ and nifH genes were examined for all possible combinations of the investigated species of the Ectothiorhodospiraceae. A significant correlation between the synonymous distances in the $c b b L$ genes and those in the nifH genes was observed, with a correlation coefficient $(r)$ of 0.91 . This result is in agreement with the assumption that the synonymous substitution rate is constant for many chromosomal genes in many organisms and that it can serve as a suitable molecular indicator of their evolution (Lawrence et al., 1991).

The total numbers of substitutions used for the calculation of genetic distances for $c b b L$ and nifH allowed estimation of the ranges of genomic variation (Table 2). The intrafamily genetic distances between the $c b b L$, nifH and 
Table 2. Ranges of genetic (Kimura), synonymous (syn) and non-synonymous (non-syn) distances calculated for $16 \mathrm{~S}$ rRNA, cbbL and nifH genes of the family Ectothiorhodospiraceae

\begin{tabular}{|c|c|c|c|c|c|c|c|}
\hline \multirow[t]{2}{*}{ Range of variation } & \multirow{2}{*}{$\begin{array}{c}\begin{array}{c}16 S \text { rRNA } \\
\text { gene }\end{array} \\
\text { Kimura }\end{array}$} & \multicolumn{3}{|c|}{$c b b L$} & \multicolumn{3}{|c|}{ nifH } \\
\hline & & Kimura & syn & non-syn & Kimura & syn & non-syn \\
\hline \multicolumn{8}{|l|}{ Within: } \\
\hline Ectothiorhodospiraceae & $0.002-0.134$ & $0.003-0.414$ & $0.011-0.764$ & $0-0.197$ & $0.003-0.295$ & $0-0.727$ & $0-0.112$ \\
\hline Ectothiorhodospira & $0.009-0.042$ & $0.063-0.133$ & $0.147-0.303$ & $0.035-0.068$ & $0.003-0.144$ & $0-0.367$ & $0-0.045$ \\
\hline Thioalkalivibrio & $0.015-0.070$ & $0.032-0.228$ & $0.089-0.462$ & $0.014-0.124$ & NA & NA & NA \\
\hline Alkalispirillum mobile & $0.002-0.012$ & $0.003-0.007$ & $0.011-0.017$ & $0-0.004$ & NA & $\mathrm{NA}$ & $\mathrm{NA}$ \\
\hline \multicolumn{8}{|l|}{ Between: } \\
\hline Ect. shaposhnikovii and Ect. vacuolata & 0.011 & 0.100 & 0.220 & 0.055 & 0.010 & 0.014 & 0.004 \\
\hline Ect. mobilis and Ect. marismortui & 0.040 & 0.095 & 0.238 & 0.041 & ND & ND & ND \\
\hline Ect. shaposhnikovii and Ect. mobilis & 0.038 & 0.086 & 0.215 & 0.037 & 0.007 & 0.014 & 0.004 \\
\hline Thiorhodospira and others & $0.052-0.126$ & $0.282-0.414$ & $0.483-0.750$ & $0.142-0.197$ & $0.180-0.280$ & $0.571-0.685$ & $0.023-0.089$ \\
\hline Thioalkalispira and others & $0.075-0.133$ & $0.199-0.377$ & $0.467-0.690$ & $0.070-0.174$ & $0.170-0.285$ & $0.415-0.711$ & $0.056-0.095$ \\
\hline Ectothiorhodosinus and others & $0.033-0.123$ & $0.213-0.342$ & $0.610-0.764$ & $0.047-0.185$ & $\mathrm{NA}$ & $\mathrm{NA}$ & $\mathrm{NA}$ \\
\hline Nitrococcus and others & $0.057-0.117$ & $0.174-0.407$ & $0.433-0.724$ & $0.053-0.197$ & $\mathrm{NA}$ & $\mathrm{NA}$ & NA \\
\hline
\end{tabular}

ND, Not determined; NA, not applicable.

$16 \mathrm{~S}$ rRNA gene sequences were up to $0.414,0.295$ and 0.134 , respectively. This indicated a variability order $c b b L>n i f H>>16 \mathrm{~S}$ rRNA gene.

The highest level of sequence divergence $(0.282-0.414)$ was detected for the $c b b L$ gene of Trs. sibirica. This was due to an increase in the number of non-synonymous substitutions. Interestingly, its nifH sequence was not so divergent from the nifH genes of other members of the Ectothiorhodospiraceae (Table 2).

On the basis of DNA-DNA hybridization analysis, Ventura et al. (2000) proposed to consider Ect. vacuolata and Ectothiorhodospira marismortui as junior synonyms of Ect. shaposhnikovii and Ect. mobilis, respectively. However, the similarities of $c b b L$ gene sequences of the pairs Ect. vacuolata-Ect. shaposhnikovii and Ect. marismortui-Ect. mobilis were at the same level as for the pair Ect. shaposhnikovii-Ect. mobilis, and much higher than for different strains of Alkalispirillum mobile (Table 2). On the other hand, although the nifH gene sequences of Ect. vacuolata and Ect. shaposhnikovii were found to be very similar (0.010), the same (high) level of similarity was shown for the sequences of Ect. shaposhnikovii and Ect. mobilis (0.007). These data are in good agreement with the current taxonomy of the genus Ectothiorhodospira, where Ect. vacuolata, Ect. shaposhnikovii, Ect. marismortui and Ect. mobilis are considered as four different species, and they disagree with the reclassification proposed by Ventura et al. (2000).

\section{Nucleotide composition and codon usage of the cbbL and nifH genes}

Genes in closely related species tend to be rather similar in their $\mathrm{G}+\mathrm{C}$ content as well as in synonymous codon usage, in contrast to genes acquired by horizontal transfer, which often have atypical $\mathrm{G}+\mathrm{C}$ content and codon usage bias (Medigue et al., 1991). Therefore, it was interesting to compare the $\mathrm{G}+\mathrm{C}$ content and codon usage of the RubisCO and nitrogenase reductase genes within the family Ectothiorhodospiraceae to detect the possible role of gene transfer in their evolution.

The total $\mathrm{G}+\mathrm{C}$ content of all analysed $c b b L$ and nifH gene fragments was close to the genomic $\mathrm{G}+\mathrm{C}$ content for each species of the family (56.2-70.7 and 56.2-66.3 against 52.9$68.4 \mathrm{~mol} \%$, respectively; Table 1 ). The $\mathrm{G}+\mathrm{C}_{3}$ content (in the third position of codons) of these genes (57.7-96.9 and 75.8-93.7 mol\%) was higher than their total G + C content and the overall genomic $\mathrm{G}+\mathrm{C}$ content; it is typical of $\mathrm{G}+\mathrm{C}$-biased micro-organisms that they preferentially use $\mathrm{G}$ or $\mathrm{C}$ in the third position of the codons (Ohtaka \& Ishikawa, 1993).

The broadest intragenus range of $\mathrm{G}+\mathrm{C}$ content variation in $c b b L$ and nifH genes (62.7-68.6 and 60.9-68.0 mol\%, respectively) was found for the Halorhodospira species, consistent with the variation of their genomic $\mathrm{G}+\mathrm{C}$ content (52.9-68.4 mol\%). Among the other representatives of the Ectothiorhodospiraceae, the lowest $\mathrm{G}+\mathrm{C}$ contents of the nifH and $c b b L$ genes (less than 
$60.3 \mathrm{~mol} \%)$ were found for the monotypic genera Ectothiorhodosinus, Thioalkalispira and Thiorhodospira, and were also comparable to their overall genomic $\mathrm{G}+\mathrm{C}$ content. Interestingly, among the species of the Alkalispirillum-Alkalilimnicola group, the $\mathrm{G}+\mathrm{C}$ content of $c b b L$ differed significantly from the genomic $\mathrm{G}+\mathrm{C}$ content and was the highest in the Ectothiorhodospiraceae (up to $71.0 \mathrm{~mol} \%$ ). At the same time, the $\mathrm{G}+\mathrm{C}$ content of the nifH gene of Alkalilimnicola halodurans (which is the only representative of the Alkalispirillum-Alkalilimnicola group that has a nifH gene) was similar to the overall genomic $\mathrm{G}+\mathrm{C}$ content.

Codon usage analysis for the $c b b L$ and nifH genes was carried out on the RSCU data. Correspondence analysis of the results (Fig. 4) identified the major trends in codon usage: the horizontal axis is associated with $G+C_{3}$, whereas the vertical axis is correlated with the frequencies of codons ending in $\mathrm{C}$ or $\mathrm{U}$ versus $\mathrm{A}$ or $\mathrm{G}$ (Fennoy \& Bailey-Serres, 1993). Codon usage analysis of $c b b L$ genes is compatible with the formation of a common group on the plot for most representatives of the Ectothiorhodospiraceae: species of Ectothiorhodospira, Halorhodospira, Thioalkalivibrio and the Alkalispirillum-Alkalilimnicola group and Nitrococcus mobilis (Fig. 4). However, the codon usage of Halorhodospira abdelmalekii was similar to that of alphaproteobacteria. The $c b b L$ codon usage bias of Trs. sibirica, Thioalkalispira microaerophila and Ectothiorhodosinus mongolicus was consistent with their $\mathrm{G}+\mathrm{C}$ content bias.

The intrafamily codon usage pattern of the nifH genes on the RSCU correspondence analysis plot was similar to the pattern of the $c b b L$ genes (Fig. 4). It appears that the codon usage bias in both $c b b L$ and nifH suggests their origin from an intrafamily genome divergence rather than from lateral gene transfer.

\section{DISCUSSION}

The use of functional genes encoding key metabolic enzymes as molecular markers is becoming common practice in phylogenetic studies. The RubisCO phylogeny, in general, differs significantly from the traditional $16 \mathrm{~S}$ rRNA gene-based phylogeny of autotrophic bacteria (Watson \& Tabita, 1997). At the same time, the topology of the 'nitrogenase' tree exhibits significant correlation with reconstructions based on 'ribosomal' gene analysis (Achouak et al., 1999; Tourova et al., 2006). This divergence can be useful to resolve particular problems of the evolution of autotrophic and diazotrophic bacteria.

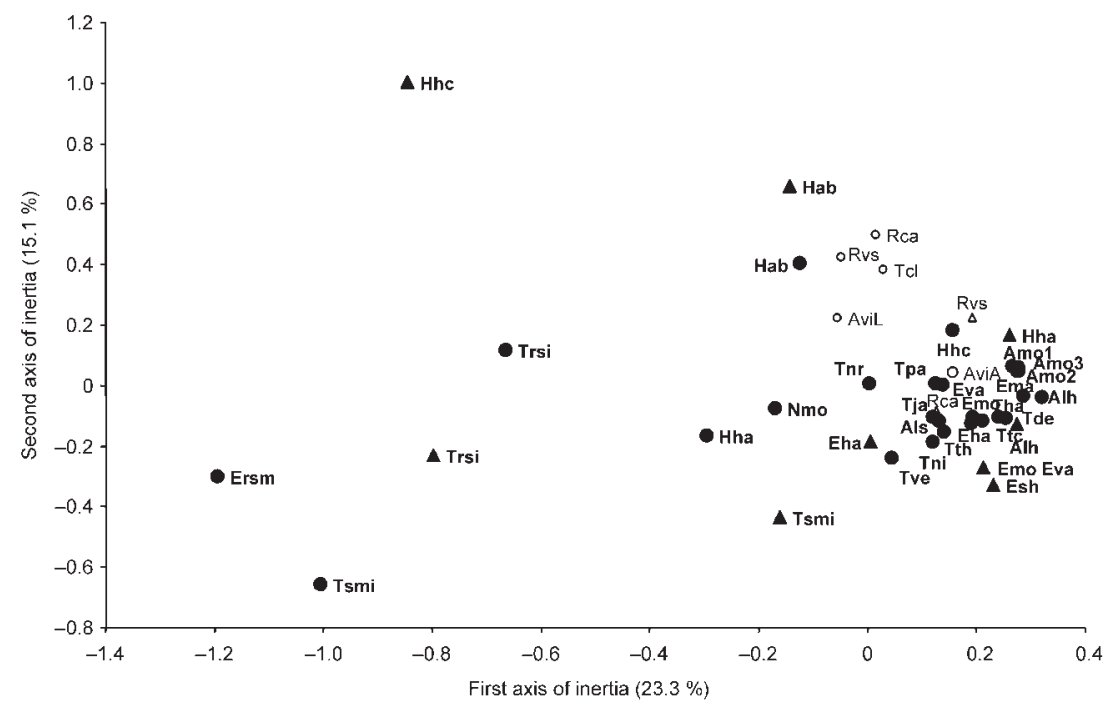

Fig. 4. Correspondence analysis of RSCU in species of the Ectothiorhodospiraceae for $c b b L$ genes of the studied group ( $\bullet$ ), $c b b L$ genes of other micro-organisms $(\bigcirc)$, nifH genes of the studied group $(\boldsymbol{\Delta})$ and nifH genes of other micro-organisms $(\triangle)$. Abbreviations: Emo, Ect. mobilis; Esh, Ect. shaposhnikovii; Eva, Ect. vacuolata; Eha, Ect. haloalkaliphila; Ema, Ect. marismortui; Hab, Hlr. abdelmalekii; Hhc, Hlr. halochloris; Hha, Hlr. halophila; Trsi, Trs. sibirica; Ersm, Ectothiorhodosinus mongolicus; Tsmi, Thioalkalispira microaerophila; Tve, Thioalkalivibrio versutus; Tde, Thioalkalivibrio denitrificans; Tth, Thioalkalivibrio thiocyanoxidans; Tha, Thialkalivibrio halophilus; Tja, Thioalkalivibrio jannaschii; Tnr, Thialkalivibrio nitratireducens; Tni, Thioalkalivibrio nitratis; Tpa, Thioalkalivibrio paradoxus; Ttc, Thialkalivibrio thiocyanodenitrificans; Amo1, Alkalispirillum mobile AGDZ; Amo2, Alkalispirillum mobile Z-0008; Amo3, Alkalispirillum mobile ALPs2; Aha, Alkalilimnicola halodurans; Als, Alkalilimnicola sp. AHN 1; Nmo, Nitrococcus mobilis; AviA, Alc. vinosum rbcA; AviL, Alc. vinosum rbcL; Rca, Rhodobacter capsulatus; Rvs, Rhodovulum sulfidophilum; Tcl, Thioclava pacifica. Genes are plotted at their co-ordinates on the two axes produced by the analysis; the axes are described in Results. 
The $c b b L_{-}$, nifH- and $16 \mathrm{~S}$ rRNA gene-based trees reconstructed in this study for the species of the Ectothiorhodospiraceae were not highly congruent in their branching patterns. While all species formed a monophyletic clade in the 'ribosomal' tree, in the 'RubisCO' and 'nitrogenase' trees, this clade disintegrated into a number of broadly distributed clusters and branches. However, most of the differences between the trees were in areas of low bootstrap values. The low resolution of the deep branches in the $c b b L$ - and nifH-based trees may be due to accelerated rates of sequence divergence or poor representation of the taxa in databases in comparison with $16 \mathrm{~S}$ rRNA gene sequences. Therefore, considering the similar codon usage in the $c b b L$ and nifH genes, these phylogenetic data may be regarded as evidence of the monophyletic origin of most $c b b L$ and nifH genes within the family Ectothiorhodospiraceae.

Nevertheless, in some cases, the 'RubisCO'-based trees showed that relationships inside the Ectothiorhodospiraceae were inconsistent both with 'ribosomal' phylogeny and phenotypic properties. This may be due to either inaccuracy in the $c b b L$ trees (phylogenetic construction bias) or occurrence of lateral gene transfer. The example is the phylogenetic position of Trs. sibirica: in both 'RubisCO' trees, it rooted with some alphaproteobacteria while, in the 'ribosomal' tree and in the 'nitrogenase' amino-acid-based tree, it clustered together with the species of the Ectothiorhodospiraceae. However, the G $+\mathrm{C}$ content of the $c b b L$ gene of Trs. sibirica (58.9 mol\%) was comparable to that of the nifH gene $(56.2 \mathrm{~mol} \%)$ and the total genome (56.7 $\mathrm{mol} \%$ ), but was significantly lower than in the available alphaproteobacterial gene sequences (64.3$65.8 \mathrm{~mol} \%)$. The codon usage patterns of nifH and $c b b L$ of Trs. sibirica were also similar and differed from the alphaproteobacterial pattern (Fig. 4). Therefore, the unusual rooting of Trs. sibirica on the 'RubisCO' tree might originate from a higher rate of non-synonymous nucleotide substitutions rather than from lateral gene transfer.

The $c b b L$ phylogeny of the cluster including the chemolithoautotrophic sulfur-oxidizers Thioalkalivibrio nitratireducens and Thioalkalivibrio paradoxus and the nitrifier Nitrococcus mobilis is of particular interest. Phylogenetic analysis of both nucleotide and amino acid sequences showed that the RubisCO genes in this group have a common origin different from the origin of the analogous genes in other species of the family. These three species clustered with strong bootstrap support with one of the duplicated $c b b L$ genes of the purple sulfur bacterium Alc. vinosum, a member of the family Chromatiaceae. One of the possible evolutionary mechanisms that could have taken place in this case is lateral gene transfer, which has presumably played a significant role in the evolution of the genes belonging to the RubisCO family (Delwiche \& Palmer, 1996; Watson \& Tabita, 1997). Similarities in codon usage (Fig. 4) and $\mathrm{G}+\mathrm{C}$ content between the duplicated $c b b L$ genes of Alc. vinosum (65.1-66.3 mol\%) and genes of Thioalkalivibrio and Nitrococcus (63.9$66.5 \mathrm{~mol} \%$ ) do not contradict this suggestion. Interestingly, both of these Thioalkalivibrio species are morphologically similar to Allochromatium (large coccoid rods with sulfur inclusions) but strikingly different from the other Thioalkalivibrio species and members of the Ectothiorhodospiraceae in general (vibrio/spirilla that deposit elemental sulfur outside the cell). It is noteworthy that the formation of intracellular sulfur globules is a characteristic feature of members of the Chromatiaceae and Allochromatium in particular. Moreover, biochemical and recent genetic studies have demonstrated that a reverse dissimilatory sulfite reductase complex encoded by a large gene cluster is responsible for further oxidation of intracellular sulfur to sulfate (Dahl et al., 2005). Preliminary tests with primers specific for the Alc. vinosum reverse $d s r$ gene complex indicated the presence of some of the genes in Thioalkalivibrio nitratireducens and Thioalkalivibrio paradoxus (C. Dahl, personal communication). Cells of Nitrococcus mobilis also have a spherical shape. The tubular membranes of Nitrococcus mobilis are quite similar to the internal photosynthetic membrane system of Thiococcus pfennigii, a member of the Chromatiaceae (Imhoff, 2005). These observations may indicate that there was an exchange of important genetic information between these different types of autotrophic bacteria. This case is just one of many examples of inconsistency between the taxonomic position determined on the basis of 16S rRNA gene sequences and other essential characteristics of the organism in question. Taking into consideration the striking structural similarity between species of the Chromatiaceae and these three representatives of the Ectothiorhodospiraceae (Thioalkalivibrio nitratireducens, Thioalkalivibrio paradoxus and Nitrococcus mobilis), it might be speculated that gene transfer between these phylogenetically distant (based on $16 \mathrm{~S}$ rRNA gene analysis) organisms might have involved not only individual genes (i.e. $c b b L$ ) but also genetic blocks. The alternative and simpler assumption may be lateral transfer of the 16S rRNA gene. Although it is generally accepted that this is a rare event, such a possibility cannot be excluded (Tourova, 2003).

The possibility of chemolithoheterotrophic aerobic growth has been shown for many representatives of the purple sulfur bacteria, from both the Chromatiaceae and the Ectothiorhodospiraceae, and some members of the Chromatiaceae can grow chemolithoautotrophically in the presence of oxygen, as colourless sulfur bacteria do (Kondratieva et al., 1976; Kämpf \& Pfennig, 1980). In the case of Thioalkalivibrio species, which are currently classified as members of the Ectothiorhodospiraceae, it might be speculated that these alkaliphilic, aerobic, sulfuroxidizing, chemolithoautotrophic bacteria represent direct aerobic descendants of the purple sulfur bacteria that have lost the genes responsible for photosynthesis. The results of the $c b b L$ gene analysis did not contradict this suggestion as a whole, but they demonstrated that the putative 
phototrophic ancestors might be different for some groups of Thioalkalivibrio species.

The genera Alkalispirillum and Alkalilimnicola were originally described as non-phototrophic, aerobic, heterotrophic relatives of the Ectothiorhodospira-Halorhodospira group. However, it has been shown recently that some novel strains and the type strain of Alkalilimnicola halodurans are capable of lithoautotrophy and have $c b b L$ genes (Oremland et al., 2002; Sorokin et al., 2006). The $c b b L$-based phylogenetic trees and codon usage analysis confirmed the relatedness of the AlkalispirillumAlkalilimnicola group to the Ectothiorhodospiraceae. However, in spite of the high DNA-DNA relatedness and the 16S rRNA gene and $c b b L$ sequence similarity, they are significantly different in details of their autotrophic metabolism (Oremland et al., 2002; Sorokin et al., 2006). For example, hydrogen-based autotrophy was found only in two strains and could be lost easily during cultivation (Sorokin et al., 2006). This might indicate the location of the RubisCO genes on a plasmid (which may be lost during heterotrophic growth). Such a loss may be a reason for the absence (temporary or constant) of the $c b b L$ genes in the genome of the type strain of Alkalispirillum mobile.

The absence of RubisCO genes in Arhodomonas aquaeolei was in accordance with the original description of this bacterium as an obligate heterotroph (Adkins et al., 1993).

Nitrogen fixation is considered to be a characteristic property of purple sulfur bacteria, including the Ectothiorhodospiraceae (Moshkovskii et al., 1971; Imhoff, 2005). Moreover, nitrogenase-mediated hydrogen production was shown for representatives of this group (Chadwick \& Irgens, 1991; Tsuihiji et al., 2006). Therefore, it is not surprising that $n i f H$ genes were detected in all phototrophic members of the Ectothiorhodospiraceae with the sole exception of Ers. mongolicus. This bacterium was isolated from a habitat enriched in organic compounds and had only a weak capacity for photoautotrophic growth (Gorlenko et al., 2004); growth under such conditions might also be accompanied by a loss of the nitrogenase genes. Since many members of the Ectothiorhodospiraceae do not have this gene and the topologies of the nifH-based trees correlate only partially with the topology of the $16 \mathrm{~S}$ rRNA gene tree, nifH does not seem to be suitable for basic phylogenetic assessments. However, its analysis may help to understand the relationship of these bacteria, especially over small phylogenetic distances.

The occurrence of nifH genes in the aerobic chemotrophic Alkalilimnicola halodurans and Thioalkalispira microaerophila is more difficult to interpret. Although analysis of these nifH genes confirmed their relatedness to nifH of other members of the Ectothiorhodospiraceae, measurement of nitrogenase activity in Alkalilimnicola halodurans and Thioalkalispira microaerophila is necessary in order to confirm whether these genes are functional. Several examples of ancient altered genes ('pseudogenes'), which can become non-functional but may still retain sufficient similarity to functional genes, are well documented (Ochman \& Davalos, 2006).

The conclusion that most of the $c b b L$ and nifH genes within the family Ectothiorhodospiraceae have a monophyletic origin allows them to be used them for analyses of microbial communities in situ. The substantial limitation of in situ analyses based on functional genes is the poor representation of many taxa (including the Ectothiorhodospiraceae) in databases. This limits identification of the obtained sequences or leads to their misidentification. The database obtained in this study could provide good support for in situ studies (for example, in haloalkaline lakes, where members of the Ectothiorhodospiraceae thrive).

In general, molecular phylogenies based on a single gene may be misleading, because of the complexity of the evolutionary process. Thus, data from several genes encoding different cellular functions are more suitable for realistic phylogenetic reconstructions.

\section{ACKNOWLEDGEMENTS}

This work was supported by the Russian Foundation for Basic Research (grant 05-04-48064), by an NWO-RFBR grant (047.011.2004.010) and by the Program on Molecular and Cell Biology of the RAS.

\section{REFERENCES}

Achouak, W., Normand, P. \& Heulin, T. (1999). Comparative phylogeny of rrs and nifH genes in the Bacillaceae. Int $J$ Syst Bacteriol 49, 961-967.

Adkins, J. P., Madigan, M. T., Mandelco, L., Woese, C. R. \& Tanner, R. S. (1993). Arhodomonas aquaeolei gen. nov., sp. nov., an aerobic, halophilic bacterium isolated from a subterranean brine. Int J Syst Bacteriol 43, 514-520.

Banciu, H., Sorokin, D. Y., Galinski, E. A., Muyzer, G., Kleerebezem, R. \& Kuenen, J. G. (2004). Thialkalivibrio halophilus sp. nov., a novel obligately chemolithoautotrophic, facultatively alkaliphilic, and extremely salt-tolerant, sulfur-oxidizing bacterium from a hypersaline alkaline lake. Extremophiles 8, 325-334.

Boulygina, E. S., Kuznetsov, B. B., Marusina, A. I., Tourova, T. P., Kravchenko, I. K., Bykova, S. A., Kolganova, T. V. \& Galchenko, V. F. (2002). A study of nucleotide sequences of nifH genes of some methanotrophic bacteria. Microbiology (English translation of Mikrobiologiia) 71, 425-432.

Bryantseva, I., Gorlenko, V. M., Kompantseva, E. I., Imhoff, J. F., Suling, J. \& Mityushina, L. (1999). Thiorhodospira sibirica gen. nov., sp. nov., a new alkaliphilic purple sulfur bacterium from a Siberian soda lake. Int J Syst Bacteriol 49, 697-703.

Chadwick, L. J. \& Irgens, R. L. (1991). Hydrogen gas production by an Ectothiorhodospira vacuolata strain. Appl Environ Microbiol 57, 594-596.

Dahl, C., Engels, S., Pott-Sperling, A. S., Schulte, A., Sander, J., Lübbe, Y., Deuster, O. \& Brune, D. C. (2005). Novel genes of the $d s r$ gene cluster and evidence for close interaction of Dsr proteins during sulfur oxidation in the phototrophic sulfur bacterium Allochromatium vinosum. J Bacteriol 187, 1392-1404.

Dauga, C. (2002). Evolution of the gyrB gene and the molecular phylogeny of Enterobacteriaceae: a model molecule for molecular systematic studies. Int J Syst Evol Microbiol 52, 531-547. 
Delwiche, C. F. \& Palmer, J. D. (1996). Rampant horizontal transfer and duplication of RubisCO genes in eubacteria and plastids. Mol Biol Evol 13, 873-882.

Felsenstein, J. (1981). Evolutionary trees from DNA sequences: a maximum likelihood approach. J Mol Evol 17, 368-376.

Felsenstein, J. (1993). PHYLIP (phylogeny inference package), version 3.53c. Distributed by the author. Department of Genome Sciences, University of Washington, Seattle, USA.

Fennoy, S. L. \& Bailey-Serres, J. (1993). Synonymous codon usage in Zea mays L. nuclear genes is varied by levels of C and G-ending codons. Nucleic Acids Res 21, 5294-5300.

Fitch, W. M. (1971). Toward defining the course of evolution: minimum change for a specific tree topology. Syst Zool 20, 406-416.

Fitch, W. M. \& Margoliash, E. (1967). Construction of phylogenetic trees. Science 155, 279-284.

Gorlenko, V. M., Bryantseva, I. A., Panteleeva, E. E., Tourova, T. P., Kolganova, T. V., Makhneva, Z. K. \& Moskalenko, A. A. (2004). Ectothiorhodosinus mongolicum gen. nov., sp. nov., a new purple bacterium from a soda lake in Mongolia. Microbiology (English translation of Mikrobiologiia) 73, 66-73.

Hoeft, S. E., Switzer Blum, J., Stolz, J. F., Tabita, F. R., Witte, B., King, G. M., Santini, J. M. \& Oremland, R. S. (2007). Alkalilimnicola ehrlichii sp. nov., a novel arsenite-oxidizing, haloalkaliphilic gammaproteobacterium capable of chemoautotrophic or heterotrophic growth with nitrate or oxygen as the electron acceptor. Int J Syst Evol Microbiol 57, 504-512.

Holmes, D. E., Nevin, K. P. \& Lovley, D. R. (2004). Comparison of $16 \mathrm{~S}$ rRNA, nifD, recA, gyrB, rpoB and fusA genes within the family Geobacteraceae fam. nov. Int J Syst Evol Microbiol 54, 1591-1599.

Imhoff, J. F. (2005). Family II. Ectothiorhodospiraceae Imhoff. In Bergey's Manual of Systematic Bacteriology, 2nd edn, vol. 2, part B, The Gammaproteobacteria, pp. 41-52. Edited by D. J. Brenner, N. R. Krieg, J. T. Staley \& G. M. Garrity. New York: Springer.

Imhoff, J. F. (2006). The family Ectothiorhodospiraceae. In The Prokaryotes: a Handbook on the Biology of Bacteria, 3rd edn, vol. 6, pp. 874-886. Edited by M. Dworkin, S. Falkow, E. Rosenberg, K. H. Schleifer \& E. Stackebrandt. New York: Springer.

Imhoff, J. F. \& Süling, J. (1996). The phylogenetic relationship among Ectothiorhodospiraceae. A re-evaluation of their taxonomy on the basis of $16 \mathrm{~S}$ rDNA analyses. Arch Microbiol 165, 106-113.

Kämpf, C. \& Pfennig, N. (1980). Capacity of Chromatiaceae for chemotrophic growth. Specific respiration rates of Thiocystis violacea and Chromatium vinosum. Arch Microbiol 127, 125-135.

Kimura, M. (1980). A simple method for estimating evolutionary rates of base substitutions through comparative studies of nucleotide sequences. J Mol Evol 16, 111-120.

Kondratieva, E. N., Zhukov, V. G., Ivanovsky, R. N., Petushkova, U. P. \& Monosov, E. Z. (1976). The capacity of phototrophic sulfur bacterium Thiocapsa roseopersicina for chemosynthesis. Arch Microbiol 108, 287-292.

Lawrence, J. G., Hartl, D. L. \& Ochman, H. (1991). Molecular considerations in the evolution of bacterial genes. J Mol Evol 33, 241-250.

Marusina, A. I., Boulygina, E. S., Kuznetsov, B. B., Tourova, T. P., Kravchenko, I. K. \& Gal'chenko, V. F. (2001). A system of oligonucleotide primers for the amplification of nifH genes of different taxonomic groups of prokaryotes. Microbiology (English translation of Mikrobiologiia) 70, 73-78.

Medigue, C., Rouxel, T., Vigier, P., Henaut, A. \& Danchin, A. (1991). Evidence for horizontal gene transfer in Escherichia coli speciation. J Mol Biol 222, 851-856.
Moshkovskii, lu. Sh., Uspenskaia, N. la. \& Mardanian, S. S. (1971). Effect of nitrogen source in the culture medium on the capability for nitrogen fixation and the Mossbauer spectra of Ectothiorhodospira shaposhnikovii cells. Biofizika 16, 933-936 (in Russian).

Nei, M. \& Gojobori, T. (1986). Simple methods for estimating the numbers of synonymous and nonsynonymous nucleotide substitutions. Mol Biol Evol 3, 418-426.

Ochman, H. \& Davalos, L. M. (2006). The nature and dynamics of bacterial genomes. Science 311, 1730-1733.

Ohtaka, C. \& Ishikawa, H. (1993). Accumulation of adenine and thymine in groE-homologous operon of an intracellular symbiont. J Mol Evol 36, 121-126.

Oremland, R. S., Hoeft, S. E., Santini, J. M., Bano, N., Hollibaugh, R. A. \& Hollibaugh, J. T. (2002). Anaerobic oxidation of arsenite in Mono Lake water and by a facultative, arsenite-oxidizing chemoautotroph, strain MLHE-1. Appl Environ Microbiol 68, 4795-4802.

Rijkenberg, M. J., Kort, R. \& Hellingwerf, K. J. (2001). Alkalispirillum mobile gen. nov., spec. nov., an alkaliphilic non-phototrophic member of the Ectothiorhodospiraceae. Arch Microbiol 175, 369-375.

Saitou, N. \& Nei, M. (1987). The neighbor-joining method: a new method for reconstructing phylogenetic trees. Mol Biol Evol 4, 406-425.

Sambrook, J., Fritsch, E. F. \& Maniatis, T. (1989). Molecular Cloning: a Laboratory Manual, 2nd edn. Cold Spring Harbor, NY: Cold Spring Harbor Laboratory.

Sorokin, D. Y., Lysenko, A. M., Mityushina, L. L., Tourova, T. P., Jones, B. E., Rainey, F. A., Robertson, L. A. \& Kuenen, G. J. (2001). Thioalkalimicrobium aerophilum gen. nov., sp. nov. and Thioalkalimicrobium sibiricum sp. nov., and Thioalkalivibrio versutus gen. nov., sp. nov., Thioalkalivibrio nitratis sp. nov. and Thioalkalivibrio denitrificans sp. nov., novel obligately alkaliphilic and obligately chemolithoautotrophic sulfur-oxidizing bacteria from soda lakes. Int $J$ Syst Evol Microbiol 51, 565-580.

Sorokin, D. Y., Tourova, T. P., Lysenko, A. M., Mityushina, L. L. \& Kuenen, J. G. (2002a). Thioalkalivibrio thiocyanoxidans sp. nov. and Thioalkalivibrio paradoxus sp. nov., novel alkaliphilic, obligately autotrophic, sulfur-oxidizing bacteria capable of growth on thiocyanate, from soda lakes. Int J Syst Evol Microbiol 52, 657-664.

Sorokin, D. Y., Gorlenko, V. M., Tourova, T. P., Tsapin, A. I., Nealson, K. H. \& Kuenen, G. J. (2002b). Thioalkalimicrobium cyclicum sp. nov. and Thioalkalivibrio jannaschii sp. nov., novel species of haloalkaliphilic, obligately chemolithoautotrophic sulfur-oxidizing bacteria from hypersaline alkaline Mono Lake (California). Int J Syst Evol Microbiol 52, 913-920.

Sorokin, D. Y., Tourova, T. P., Kolganova, T. V., Sjollema, K. A. \& Kuenen, J. G. (2002c). Thioalkalispira microaerophila gen. nov., sp. nov., a novel lithoautotrophic, sulfur-oxidizing bacterium from a soda lake. Int J Syst Evol Microbiol 52, 2175-2182.

Sorokin, D. Y., Zhilina, T. N., Lysenko, A. M., Tourova, T. P. \& Spiridonova, E. M. (2006). Metabolic versatility of haloalkaliphilic bacteria from soda lakes belonging to the AlkalispirillumAlkalilimnicola group. Extremophiles 10, 213-220.

Spiridonova, E. M., Berg, I. A., Kolganova, T. V., Ivanovskii, R. N., Kuznetsov, B. B. \& Tourova, T. P. (2004). An oligonucleotide primer system for amplification of the ribulose-1,5-bisphosphate carboxylase/oxygenase genes of bacteria of various taxonomic groups. Microbiology (English translation of Mikrobiologiia) 73, 377-387.

Teske, A., Alm, E., Regan, J. M., Toze, S., Rittmann, B. E. \& Stahl, D. A. (1994). Evolutionary relationship among ammonia- and nitriteoxidizing bacteria. J Bacteriol 176, 6623-6630.

Thompson, J. D., Higgins, D. G. \& Gibson, T. J. (1994). CLUSTAL W: improving the sensitivity of progressive multiple sequence alignment 
through sequence weighting, position-specific gap penalties and weight matrix choice. Nucleic Acids Res 22, 4673-4680.

Tourova, T. P. (2003). Copy number of ribosomal operons in prokaryotes and its effect on phylogenetic analyses. Microbiology (English translation of Mikrobiologiia) 72, 389-402.

Tourova, T. P., Spiridonova, E. M., Berg, I. A., Kuznetsov, B. B. \& Sorokin, D. Yu. (2005). Phylogeny of ribulose-1,5-bisphosphate carboxylase/oxygenase genes in haloalkaliphilic obligately autotrophic sulfur-oxidizing bacteria of the genus Thioalkalivibrio. Microbiology (English translation of Mikrobiologiia) 74, 321-328.

Tourova, T. P., Spiridonova, E. M., Slobodova, N. V., Boulygina, E. S., Keppen, O. I., Kuznetsov, B. B. \& Ivanovsky, R. N. (2006). Phylogeny of anoxygenic filamentous phototrophic bacteria of the family Oscillochloridaceae as inferred from comparative analyses of the rrs, $c b b L$, and nifH genes. Microbiology (English translation of Mikrobiologiia) 75, 192-200.

Tsuihiji, H., Yamazaki, Y., Kamikubo, H., Imamoto, Y. \& Kataoka, M. (2006). Cloning and characterization of nif structural and regulatory genes in the purple sulfur bacterium, Halorhodospira halophila. J Biosci Bioeng 101, 263-270.
Van de Peer, Y. \& De Wachter, R. (1994). TREECON for Windows: a software package for the construction and drawing of evolutionary trees for the Microsoft Windows environment. Comput Appl Biosci 10, 569-570.

Ventura, S., Viti, C., Pastorelli, R. \& Giovannetti, L. (2000). Revision of species delineation in the genus Ectothiorhodospira. Int J Syst Evol Microbiol 50, 583-591.

Watson, G. M. \& Tabita, F. R. (1997). Microbial ribulose-1,5bisphosphate carboxylase/oxygenase: a molecule for phylogenetic and enzymological investigation. FEMS Microbiol Lett 146, 13-22.

Watson, S. W. \& Waterbury, J. B. (1971). Characteristics of two marine nitrite oxidizing bacteria, Nitrospira gracilis nov. gen. nov. sp. and Nitrococcus mobilis nov. gen. nov. sp. Arch Mikrobiol 77, 203-230.

Yakimov, M. M., Giuliano, L., Chernikova, T. N., Gentile, G., Abraham, W.-R., Lünsdorf, H., Timmis, K. N. \& Golyshin, P. N. (2001). Alcalilimnicola halodurans gen. nov., sp. nov., an alkaliphilic, moderately halophilic and extremely halotolerant bacterium, isolated from sediments of soda-depositing Lake Natron, East Africa Rift Valley. Int J Syst Evol Microbiol 51, 2133-2143. 